\title{
A CHARACTERIZATION OF COMPLETE RIEMANNIAN MANIFOLDS MINIMALLY IMMERSED IN THE UNIT SPHERE*
}

\author{
QING-MING CHENG
}

\section{\$1. Introduction}

Let $M^{n}$ be an $n$-dimensional Riemannian manifold minimally immersed in the unit sphere $S^{n+p}(1)$ of dimension $n+p$. When $M^{n}$ is compact, Chern, do Carmo and Kobayashi [1] proved that if the square $\|h\|^{2}$ of length of the second fundamental form $h$ in $M^{n}$ is not more than $\frac{n}{2-1 / p}$, then either $M^{n}$ is totally geodesic, or $M^{n}$ is the Veronese surface in $S^{4}(1)$ or $M^{n}$ is the Clifford torus $S^{k}(\sqrt{k / n}) \times S^{n-k}(\sqrt{(n-k) / n})$ in $S^{n+1}(1)(0<k<n)$.

In this paper, we generalize the results due to Chern, do Carmo and Kobayashi [1] to complete Riemannian manifolds.

Acknowledgement. The author would like to express his gratitude to Professors K. Shiohama and H. Nakagawa for their valuable suggestions.

\section{§2. Preliminaries}

Let $M^{n}$ be an $n$-dimensional Riemannian manifold which is minimally immersed in the unit sphere $S^{n+p}(1)$ of dimension $n+p$. Then the second fundamental form $h$ of the immersion is given by $h(X, Y)=\tilde{\nabla}_{X} Y-\nabla_{X} Y$ and it satisfies $h(X, Y)=h(Y, X)$, where $\tilde{\nabla}$ and $\nabla$ denote the covariant differentiations on $S^{n+p}(1)$ and $M^{n}$ respectively, $X$ and $Y$ are vector fields on $M^{n}$. We choose a local field of orthonormal frames $e_{1}, \ldots, e_{n+p}$ in $S^{n+p}(1)$ such that, restricted to $M^{n}$, the vectors $e_{1}, \ldots, e_{n}$ are tangent to $M^{n}$. We use the following convention on the range of indices unless otherwised stated: $A, B, C, \cdots=1,2, \ldots$, $n+p ; i, j, k, \cdots=1,2,3, \ldots, n ; \alpha, \beta, \cdots=n+1, \ldots, n+p$. We agree the

Received November 21, 1988.

* The project Supported by NNSFC. 
repeated indices under a summation sign without indication are summed over the respective range. With respect to the frame field of $S^{n+p}(1)$ chosen above, let $\omega_{1}, \ldots, \omega_{n+p}$ be the dual frame. Then the structure equations of $S^{n+p}(1)$ are given by

$$
\begin{aligned}
& d \omega_{A}=\sum \omega_{A B} \wedge \omega_{B}, \omega_{A B}+\omega_{B A}=0, \\
& d \omega_{A B}=\sum \omega_{A C} \wedge \omega_{C B}-\omega_{A} \wedge \omega_{B} .
\end{aligned}
$$

Restricting these forms to $M^{n}$, we have the structure equations of the immersion:

$$
\begin{aligned}
& \omega_{\alpha}=0, \\
& \omega_{i \alpha}=\sum h_{i j}^{\alpha} \omega_{j}, \quad h_{i j}=h_{j i}, \\
& d \omega_{i}=\sum \omega_{i j} \wedge \omega_{j}, \quad \omega_{i j}+\omega_{j i}=0, \\
& d \omega_{i j}=\sum \omega_{i k} \wedge \omega_{k j}-\Omega_{i j}, \quad \Omega_{i j}=\frac{1}{2} \sum R_{i j k l} \omega_{k} \wedge \omega_{l}, \\
& R_{i j k l}=\left(\delta_{i k} \delta_{j l}-\delta_{i l} \delta_{j k}\right)+\sum\left(h_{i k}^{\alpha} h_{j l}^{\alpha}-h_{l l}^{\alpha} h_{j k}^{\alpha}\right), \\
& d \omega_{\alpha \beta}=\sum \omega_{\alpha \gamma} \wedge \omega_{r \beta}-\Omega_{\alpha \beta}, \quad \Omega_{\alpha \beta}=\frac{1}{2} \sum R_{\alpha \beta i j} \omega_{i} \wedge \omega_{j}, \\
& R_{\alpha \beta i j}=\sum\left(h_{i k}^{\alpha} h_{k j}^{\beta}-h_{j k}^{\alpha} h_{k i}^{\beta}\right) .
\end{aligned}
$$

Then, the second fundamental form $h$ can be written as

$$
h\left(e_{i}, e_{j}\right)=\sum h_{i j}^{\alpha} e_{\alpha} .
$$

We denote the square of the length of $h$ by $\|h\|^{2}$. Then $\|h\|^{2}$ is intrinsic and given by $\|h\|^{2}=n(n-1)-R$, where $R$ is the scalar curvature. If we define $h_{i j k}^{\alpha}$ by

$$
\sum h_{i j k}^{\alpha} \omega_{k}=d h_{i j}^{\alpha}+\sum h_{j k}^{\alpha} \omega_{k i}+\sum h_{i k}^{\alpha} \omega_{k j}+\sum h_{i j}^{\beta} \omega_{\beta \alpha},
$$

then, from (2.2), (2.3) and (2.4), we have $h_{i j k}^{\alpha}=h_{i k j}^{\alpha}$.

In this paper, we denote the image of the immersion by $M^{n}$ for simplicity.

Lemma 1 (cf. [2]). Let $M^{n}$ be a Riemannian manifold minimally immersed in $S^{n+p}(1)$. Then for any unit vector $v$ on $M^{n}$,

$$
\operatorname{Ric}(v, v) \geq \frac{n-1}{n}\left(n-\|h\|^{2}\right),
$$

where $\operatorname{Ric}(v, v)$ denotes the Ricci curvature in the $v$ direction. 
Lemma 2 (cf. [3]). Let $M^{n}$ be a complete Riemannian manifold with Ricci curvature bounded from below. Let $f$ be a $C^{2}$-function bounded from above on $M^{n}$, then for all $\varepsilon>0$, there exists a point $x$ in $M^{n}$ such that at $x$,

$$
\begin{gathered}
f(x)>\sup f-\varepsilon, \\
\|\nabla f\|<\varepsilon, \\
\Delta f<\varepsilon .
\end{gathered}
$$

\section{§3. Main results}

THEOREM 1. Let $M^{n}$ be an $n$-dimensional complete Riemannian manifold minimally immersed in the unit sphere $S^{n+p}(1)$ of dimension $n+p$. Then either $M^{n}$ is totally geodesic and $M^{n}$ is globally isometric to $S^{\mathrm{n}}(1)$, or inf $R \leq n(n-1)-$ $\frac{n}{2-1 / p}$

Proof. Following the computation in [1], we have

$$
\frac{1}{2} \Delta\|h\|^{2}=\sum\left(h_{i j k}^{\alpha}\right)^{2}-K_{N}-L_{N}+n\|h\|^{2} .
$$

Because

$$
\sum_{i j}\left(\sum_{k}\left(h_{i k}^{\alpha} h_{k j}^{\beta}-h_{j k}^{\alpha} h_{k i}^{\beta}\right)\right)^{2} \leq 2 \sum_{i j}\left(h_{i j}^{\alpha}\right)^{2} \sum_{i j}\left(h_{i j}^{\beta}\right)^{2}
$$

we get

$$
\begin{aligned}
K_{N} & =\sum\left(\sum_{k}\left(h_{i k}^{\alpha} h_{k j}^{\beta}-h_{\jmath k}^{\alpha} h_{k i}^{\beta}\right)\right)^{2} \\
& \leq 2 \sum_{\alpha \neq \beta} \sum_{i j}\left(h_{i j}^{\alpha}\right)^{2} \sum_{i j}\left(h_{i j}^{\beta}\right)^{2}=2\|h\|^{4}-2 \sum\left(\sum_{i j}\left(h_{i j}^{\alpha}\right)^{2}\right)^{2} .
\end{aligned}
$$

(3.1) and (3.3) imply

$$
\frac{1}{2} \Delta\|h\|^{2} \geq\|h\|^{2}\left[n-\left(2-\frac{1}{p}\right)\|h\|^{2}\right] .
$$

1) If inf $R \leq n(n-1)-\frac{n}{2-1 / p}$, then Theorem 1 is true.

2) If $\inf R>n(n-1)-\frac{n}{2-1 / p}$, then $R>n(n-1)-\frac{n}{2-1 / p}$. We have 


$$
\|h\|^{2}=n(n-1)-R<\frac{n}{2-1 / p} .
$$

Hence, $\|h\|^{2}$ is bounded. According to Lemma 1 , we know that the Ricci curvature of $M^{n}$ is bounded from below. In fact, from (2.12) and (3.5), we have, for any unit vector $v$,

$$
\operatorname{Ric}(v, v) \geq \frac{n-1}{n}\left(n-\|h\|^{2}\right) \geq(n-1)\left[1-\frac{1}{2-1 / p}\right] .
$$

We define $f=\|h\|^{2}, F=(f+a)^{1 / 2}$ (where $a>0$ is any positive constant number). $F$ is bounded because $\|h\|^{2}$ is bounded.

$$
\begin{gathered}
d F=\frac{1}{2}(f+a)^{-1 / 2} d f, \\
\Delta F=\frac{1}{2}\left[-\frac{1}{2}(f+a)^{-3 / 2}\|d f\|^{2}+(f+a)^{-1 / 2} \Delta f\right] \\
=\frac{1}{2}\left[-2\|d F\|^{2}+\Delta f\right](f+a)^{-1 / 2}=\frac{1}{2 F}\left[-2\|d F\|^{2}+\Delta f\right] .
\end{gathered}
$$

Hence, $F \Delta F=-\|d F\|^{2}+\frac{1}{2} \Delta f$, namely,

$$
\frac{1}{2} \Delta f=F \Delta F+\|d F\|^{2} \text {. }
$$

Applying the Lemma 2 to $F$, we have for all $\varepsilon>0$, there exists a point $x$ in $M^{n}$ such that at $x$,

$$
\begin{aligned}
& \|d F(x)\|<\varepsilon, \\
& \Delta F(x)<\varepsilon, \\
& F(x)>\sup F-\varepsilon .
\end{aligned}
$$

(3.6), (3.7) and (3.8) imply

$$
\frac{1}{2} \Delta f<\varepsilon^{2}+F \varepsilon=\varepsilon(\varepsilon+F) \quad(\text { by } F>0) .
$$

We take a sequence $\left\{\varepsilon_{m}\right\}$ such that $\varepsilon_{m} \rightarrow 0(m \rightarrow \infty)$ and for all $m$, there exists a point $x_{m}$ in $M^{n}$ such that (3.7), (3.8) and (3.9) hold good. Hence, $\varepsilon_{m}\left(\varepsilon_{m}+\right.$ $\left.F\left(x_{m}\right)\right\} \rightarrow 0(m \rightarrow \infty)$ because $F$ is bounded.

On the other hand, from (3.9), 


$$
F\left(x_{m}\right)>\sup F-\varepsilon_{m} .
$$

Since $F$ is bounded, $\left\{F\left(x_{m}\right)\right\}$ is a bounded sequence, and we get

$$
F\left(x_{m}\right) \rightarrow F_{0},
$$

if necessary, we can choose subsequence. Hence,

$$
F_{0} \geq \sup F \text {. }
$$

According to the definition of supremum, we have

$$
F_{0}=\sup F \text {. }
$$

From the definition of $F$, we get

$$
f\left(x_{m}\right) \rightarrow f_{0}=\sup f \quad\left(\text { by } F_{0}=\sup F\right) .
$$

From (3.4) and (3.10), we obtain

$$
\begin{gathered}
f[n-(2-1 / p) f] \leq \frac{1}{2} \Delta f<\varepsilon^{2}+\varepsilon F, \\
f\left(x_{m}\right)\left[n-(2-1 / p) f\left(x_{m}\right)\right]<\varepsilon_{m}^{2}+\varepsilon_{m} F\left(x_{m}\right) \leq \varepsilon_{m}^{2}+\varepsilon_{m} F_{0} .
\end{gathered}
$$

Let $m \rightarrow \infty$, we have $\varepsilon_{m} \rightarrow 0, f\left(x_{m}\right) \rightarrow f_{0}$. Hence,

$$
f_{0}\left[n-(2-1 / p) f_{0}\right] \leq 0 \text {. }
$$

1) If $f_{0}=0$, we have $f=\|h\|^{2}=0$. Hence $M^{n}$ is totally geodesic, and we know that $M^{n}$ is globally isometric to $S^{n}(1)$.

2) If $f_{0}>0$, we have

$$
n-(2-1 / p) f_{0} \leq 0, \quad f_{0} \geq \frac{n}{2-1 / p}
$$

that is, $\sup \|h\|^{2} \geq \frac{n}{2-1 / p}$. From (2.15),

$$
\inf R \leq n(n-1)-\frac{n}{2-1 / p} \text {. }
$$

This completes the proof of Theorem 1.

THEOREM 2. Let $M^{n}$ be an $n$-dimensional complete Riemannian manifold minimally immersed in the unit sphere $S^{n+p}(1)$ of dimension $n+p$. If $n>1, p>1$, then either $M^{n}$ is totally geodesic and $M^{n}$ is globally isometric to $S^{n}(1)$, or $M^{n}$ is the 
Veronese surface in $S^{4}(1)$ or $\inf R<n(n-1)-\frac{n}{2-1 / p}$.

Proof. According to the proof of Theorem 1, we know

$$
\|h\|^{2}=0 \quad \text { or } \quad \sup \|h\|^{2} \geq \frac{n}{2-1 / p}
$$

1) If $\|h\|^{2}=0$, then $M^{n}$ is totally geodesic and $M^{n}$ is globally isometric to $S^{n}(1)$ from Theorem 1.

2) If $\sup \|h\|^{2} \geq \frac{n}{2-1 / p}$, then we have

$$
\inf R=n(n-1)-\sup \|h\|^{2} \leq n(n-1)-\frac{n}{2-1 / p} \text {. }
$$

When inf $R<n(n-1)-\frac{n}{2-1 / p}$, we know that Theorem 2 holds.

When inf $R=n(n-1)-\frac{n}{2-1 / p}$, we have

$$
\sup \|h\|^{2}=\frac{n}{2-1 / p} \text {. }
$$

Hence,

$$
\|h\|^{2} \leq \frac{n}{2-1 / p}
$$

According to Lemma 1 , we get, for any unit vector $v$ in $M^{n}$,

$$
\begin{aligned}
\operatorname{Ric}(v, v) & \geq \frac{n-1}{n}\left[n-\frac{n}{2-1 / p}\right] \\
& \geq(n-1)\left[1-\frac{1}{2-1 / p}\right]>0 \quad(\text { by } p>1, n>1) .
\end{aligned}
$$

From Myers' Theorem, we know that $M^{n}$ is compact. Main theorem, Corollary and theorem 3 in [1] yield $p=n=2$ and $M^{n}$ is the Veronese surface in $S^{4}(1)$. This completes the proof of Theorem 2 .

THeorem 3. Let $M^{n}$ be an $n$-dimensional connected complete Riemannian manifold immersed in the unit sphere $S^{n+1}(1)$ of dimension $n+1$. If there is a point $p$ in $M^{n}$ and a unit vector $v$ such that $\operatorname{Ric}(v, v)(p)=0$, then either $M^{n}$ is totally geodesic and $M^{n}$ is globally isometric to $S^{n}(1)$, or $M^{n}$ is locally the Clifford torus $S^{k}(\sqrt{k / n}) \times S^{n-k}(\sqrt{(n-k) / n})$ in $S^{n+1}(1)(0<k<n)$, or inf $R<n(n-2)$. 
Proof. According to Theorem 1, we know that either $M^{n}$ is totally geodesic and $M^{n}$ is globally isometric to $S^{n}(1)$, or $\inf R \leq n(n-1)-n=n(n-2)$ (from $p=1$ ).

1) If $M^{n}$ is totally geodesic or inf $R<n(n-2)$, then Theorem 3 is true.

2) If $\inf R=n(n-2)$, then $\sup \|h\|^{2}=n$. Hence, $\|h\|^{2} \leq n$. When $\|h\|^{2}$ get its maximum in $M^{n}$, that is, there is a point $p$ in $M^{n}$ such that $\|h(p)\|^{2}=\sup \|h\|^{2}$, we have $\|h\|^{2}=n$ from $E$. Hopf's Theorem. Theorem 2 of [1] implies that Theorem 3 is true. When $\|h\|^{2}<n$, we will show that it is impossible. In fact, if $\|h\|^{2}<n$, we have

$$
\operatorname{Ric}(v, v) \geq(n-1)\left(1-\frac{\|h\|^{2}}{n}\right)>0
$$

This is a contradiction.

\section{REFERENCES}

[1] S.S. Chern, M. do Carmo and S. Kobayashi, Minimal submanifolds of a sphere with second fundamental form of constant length, Functional analysis and related fields, (1970), 60-75.

[2] P.F. Leung, Minimal submanifolds in a sphere, Math. Z. 183 (1983), 86-75.

[3] S.T. Yan, Harmonic functions on complete Riemannian manifolds, Comm. Pure and Appl. Math. 28 (1975), 201-228.

Institute of Mathematics

Fudan University

Shanghai, P.R. China 